\title{
Hemoptisis relacionada a una aspergilosis pulmonar cavitaria cónica: presentación de un caso
}

\author{
Pedro Alexander Guerrero-Serrano* \\ Javier Enrique Fajardo-Rivero** \\ Martha Liliana Alarcón-Tarazona*** \\ Jonathan Alexis Duarte-Villamizar**** \\ Isabel Cristina Bolívar-Aguilera *****
}

\begin{abstract}
*Estudiante de XII semestre de medicina. Universidad Industrial de Santander. Escuela de Medicina. Facultad de Salud. Bucaramanga. Santander. Colombia.

**Médico Internista. Neumólogo. Profesor del Departamento de Medicina Interna. Universidad Industrial de Santander. Bucaramanga. Santander. Colombia.

***Médico y cirujano. Residente de 2 año Medicina Interna. Universidad Industrial de Santander. Bucaramanga. Santander. Colombia. ****Médico y cirujano. Hospital Universitario Los Comuneros. Bucaramanga. Santander. Colombia.

***** Médico y cirujano. Patóloga. Neumopatóloga. Miembro del Departamento de Patología. Universidad Industrial de Santander. Bucaramanga. Santander. Colombia.

Correspondencia: Pedro Guerrero. Dirección: Calle 29 \# 31-04 apto 102. Teléfono: 3177495502. Correo electrónico: p.guerrero19@hotmail.com.
\end{abstract}

\section{RESUMEN}

Introducción: la aspergilosis pulmonar crónica es una patología infrecuente, su prevalencia aumenta en asociación con alteraciones estructurales, esto resalta la importancia de su conocimiento teniendo en cuenta la elevada incidencia de tuberculosis en Colombia. Por el carácter angioinvasivo del hongo la posibilidad de desarrollar hemoptisis es alta, pudiendo llegar a comprometer la vida de quien la padece. Objetivo: estudiar la forma de presentación y abordaje diagnóstico de la aspergilosis y su asociación con secuelas de tuberculosis pulmonar. Presentación de caso: se presenta el caso de un paciente masculino de 44 años, con secuelas pulmonares por tuberculosis, que requirió hospitalización por hemoptisis recurrente; con hallazgos imagenológicos sugestivos de aspergilosis pulmonar, requirió estabilización clínica, embolización arterial selectiva y resección de la lesión. Conclusión: la aspergilosis es una enfermedad inusual y se requiere de la integración de aspectos clínicos e imagenológicos representativos para hacer un diagnóstico acertado. MÉD.UIS. 2017;30(1):79-86.

Palabras clave: Aspergilosis Pulmonar. Hemoptisis. Embolización terapéutica. Neumonectomía. Tuberculosis. Aspergillus.

\section{Hemoptysis related to chronic cavitary aspergillosis of the lung: case report}

\section{ABSTRACT}

Introduction: the chronic pulmonary aspergillosis is an uncommon condition, its prevalence increases in association with structural alterations, this highlights the importance of its knowledge bearing in mind the high incident of tuberculosis in Colombia. for the angioinvasive behavior of the fungus, the possibility of developing hemoptysis is high even life threatening. Objective: to study the form of presentation and diagnostic approach of the aspergilosis and his association with sequels of pulmonary tuberculosis. Case Report: we present a case of a 44-year-old masculine patient, with pulmonary sequels for tuberculosis who required hospitalization because of recurring hemoptysis; some imagenologic findings were found suggesting of pulmonary aspergillosis, he required clinical stabilization, selective arterial embolization and finally resection of the lesion. Conclusion: the aspergillosis is an unusual disease and requires integration of non-accurate clinical aspects and some representative imagenologic findings to make a proper diagnostic. MÉD.UIS. 2017;30(1):79-86.

Keywords: Pulmonary Aspergillosis. Hemoptysis. Therapeutic Embolization. Pneumonectomy. Tuberculosis. Aspergillus. 
¿Cómo citar este artículo?: Guerrero-Serrano PA, Fajardo-Rivero JE, Alarcón-Tarazona ML, Duarte-Villamizar JA, Bolívar - Aguilera IC. Hemoptisis relacionada a una aspergilosis pulmonar cavitaria cónica: presentación de un caso. MÉD.UIS. 2017;30(1):79-86.

\section{INTRODUCCIÓN}

Las especies de Aspergillus son ubicuas en la naturaleza y de distribución universal. El espectro clínico de su infección es muy amplio y dentro de este se destaca la Aspergilosis Pulmonar Cavitaria (APC) que es una enfermedad de presentación heterogénea con diferentes formas clínicas que incluyen: aspergiloma simple, Aspergilosis Pulmonar Cavitaria Crónica (APCC), aspergilosis pulmonar fibrosante crónica, aspergilosis nodular y aspergilosis invasiva subaguda necrotizante crónica o semi-invasiva, en donde el subtipo cavitario crónico es una forma frecuente de afectación ${ }^{1,2}$. Hay una tendencia hacia una mayor frecuencia en el sexo masculino y tiene una fuerte asociación con alteraciones estructurales del parénquima pulmonar, especialmente cavitaciones y bronquiectasias, entre ellas cabe mencionar la enfermedad pulmonar obstructiva crónica y por tratarse de una enfermedad frecuente en Colombia se destaca la tuberculosis pulmonar ${ }^{3}$.

La APC fue descrita como una patología fatal por primera vez en el Reino Unido en 1842 y desde entonces se han realizado varias descripciones para tratar de unificar criterios y clasificar esta patología que puede tener presentaciones clínicas variables'. (Ver Tabla 1).

La carga global de APC como consecuencia de tuberculosis pulmonar es sustancial, lo que resalta la importancia de su conocimiento, teniendo en cuenta la alta incidencia de tuberculosis pulmonar en Colombia. De esta manera, cerca del $50 \%$ de quienes han tenido tuberculosis presentan secuelas pulmonares estructurales y se ha descrito que el $22 \%$ de las personas con cambios estructurales tienen un compromiso sobreimpuesto por Aspergillus spp 4 .

No solo las alteraciones estructurales del parénquima pulmonar se catalogan como factores de riesgo para adquirir el germen, se mencionan medicamentos o condiciones preexistentes que alteran crónicamente la respuesta inmune tales como el manejo con corticoide sistémico de forma prolongada, los quimioterapéuticos o patologías como la infección por el virus de la inmunodeficiencia humana ${ }^{2,5,6}$.
La APCC en general, muestra múltiples cavidades que pueden contener o no un aspergiloma, ocasionando síntomas sistémicos y pulmonares durante al menos tres meses de seguimiento, la infección pulmonar crónica por Aspergillus puede cursar con tos y hemoptisis, sin embargo, en algunas personas la enfermedad puede ser netamente asintomática ${ }^{4}$. La afectación pulmonar se observa de forma preferencial hacia los lóbulos superiores, como lesiones únicas o múltiples?.

Para el diagnóstico se requiere de la combinación de características clínicas, hallazgos imagenológicos compatibles y de la evidencia directa o indirecta de infección por Aspergillus spp, excluyendo otras condiciones probables?. Entre los diagnósticos diferenciales se debe considerar, la histoplasmosis, paracoccidioidomicosis y coccidioidomicosis ${ }^{2}$.

Dentro de los métodos diagnósticos se puede optar por el antígeno galactomanano, el cual es útil ante la sospecha diagnóstica de infección micótica en estadios iniciales, cuando no se encuentran hallazgos clínicos o imagenológicos claros, sin embargo, la sensibilidad y especificidad varía dependiendo de el tipo de muestra a estudio, el uso conjunto de algunos esquemas antibióticos y el estadio de la enfermedad ${ }^{8}$.

El tratamiento tiene como objetivo el control de la infección, reducir o detener el daño pulmonar estructural, mejorar la calidad de vida y prevenir los episodios de hemoptisis ${ }^{6}$. La decisión de manejo médico y la duración del mismo dependen del tipo de variante de APC. Hasta ahora, con los datos disponibles, se considera que el tratamiento antifúngico provee algunos beneficios terapéuticos en caso de APC en progresión o sintomática ${ }^{7}$. No existe consenso sobre la duración del tratamiento pero se asume con respecto a la respuesta clínica del paciente en cada variante de $\mathrm{APC}^{6}$. El manejo quirúrgico es la estrategia de manejo curativo que se debe implementar en estos casos, la cual previene las complicaciones previamente descritas.

Se presenta el caso de un hombre inmunocompetente, trabajador de la piedra, con antecedente y secuelas por tuberculosis pulmonar, que tuvo síntomas 
respiratorios y constitucionales crónicos; consultó por hemoptisis masiva recurrente que derivó en una serie de intervenciones médicas en pro de su estabilización antes de realizar la resección quirúrgica de la lesión.

La aspergilosis pulmonar aumenta su incidencia de forma significativa cuando existen alteraciones estructurales del parénquima pulmonar y paralelamente aumentan las complicaciones subyacentes a esta enfermedad, entre ellas la hemoptisis que en caso de ser significativa puede poner en riesgo la vida de los pacientes que la padecen, de esta manera el presente reporte de caso busca estudiar la forma de presentación y abordaje diagnóstico de la aspergilosis y su asociación con secuelas de tuberculosis pulmonar.

Tabla 1. Críterios diagnósticos para las diferentes formas de aspergillosis pulmonar crónica.

\begin{tabular}{|c|c|}
\hline Aspergiloma simple & $\begin{array}{l}\text { Cavidad pulmonar solitaria que contiene una bola de hongos, con evidencia } \\
\text { serológica o microbiologica de Aspergillus spp. en un paciente no } \\
\text { inmnocomprometido con sintomas menores o sin síntomas y no progresión } \\
\text { radiológica por lo menos durante tres meses de observación. }\end{array}$ \\
\hline $\begin{array}{l}\text { Aspergilosis pulmonar } \\
\text { cavitaria crónica }\end{array}$ & $\begin{array}{l}\text { Uno o más cavidades pulmonares (con pared delgada o gruesa) que } \\
\text { posiblemente contenga uno o másaspergilomas o materialirregular intraluminal, } \\
\text { con evidencia serológica o microbiológica de Aspergillus spp. Con síntomas } \\
\text { pulmonares o sistémicos significativos y progresión radiológica manifiesta } \\
\text { (nuevas cavidades, aumento de los infiltrados pericavitarios o aumento de la } \\
\text { fibrosis) durante al menos tres meses de observación. }\end{array}$ \\
\hline $\begin{array}{l}\text { Aspergilosis pulmonar } \\
\text { fibrosante crónica }\end{array}$ & $\begin{array}{l}\text { Severa destrucción fibrótica de al menos dos lóbulos pulmonares que complican } \\
\text { una aspergillosis pulmonar cavitaria que conduce a una importante pérdida de } \\
\text { la función pulmonar. Destrucción severa fibrótica de un lóbulo con una cavidad } \\
\text { referido como una aspergilosis pulmonar cavitaria crónica que afecta el mismo } \\
\text { lóbulo. Por lo general, la fibrosis se manifiesta como consolidción, pero grandes } \\
\text { cavidades con fibrosis rodeandolas pueden observarse. }\end{array}$ \\
\hline Aspergilosis nodular & $\begin{array}{l}\text { Uno o más nodulos que pueden no cavitarse, se trata de una forma poco } \\
\text { frecuente de aspergilosis pulmonar crónica. Pueden imitar un tuberculoma, } \\
\text { carcinoma pulmonar, coccidioidomicosis entre otros. Su diagnóstico es } \\
\text { histológico, la invasión de téjidos no se demuestra aunque es frecuente la } \\
\text { necrosis. }\end{array}$ \\
\hline $\begin{array}{l}\text { Aspergilosis invasiva } \\
\text { subaguda, necrotizante } \\
\text { crónica o semi-invasiva }\end{array}$ & $\begin{array}{l}\text { La aspergilosis invasiva por lo general ocurre en pacientes con algún grado } \\
\text { de inmunocompromiso, se produce durante uno a tres meses, con diferentes } \\
\text { variables radiológicas que incluyen: cavitación, nódulos, consolidación que } \\
\text { puede progresar a abscesos. La biopsia muestra hifas invadiendo el téjido } \\
\text { pulmonar y los estudios microbiológicos muestran aspergillosis invasiva, } \\
\text { el antígeno galactomanano es notablemente positivo en sagre (o fluidos } \\
\text { respiratorios). }\end{array}$ \\
\hline
\end{tabular}

Fuente: Deening DW, Cadranel J, Beigelman-Aubry C, Ader F, Chakrabarti A, Blot S, at cols. Chronic pulmonary aspergillosis: rationale and clinical guidelines for diagnosis and management. Eur Respir J. 2016;47:45-68.

\section{Presentación del Caso}

Se presenta el caso de un hombre de 44 años, trabajador de la piedra, procedente de San Gil, Santander, con historia de tuberculosis pulmonar tratada en el 2008. Consultó por una semana de hemoptisis intermitente asociada a fiebre no cuantificada y escalofríos, adicionalmente, disnea de esfuerzos y pérdida de peso de $3 \mathrm{~kg}$. Tuvo un episodio puntual de hemoptisis cuantificada en $400 \mathrm{~mL}$. En el hospital en donde consultó inicialmente, le realizaron una tomografía axial del tórax que mostró una lesión cavitada apical derecha con una imagen en su interior que sugería una "bola de hongos", fue derivado para valoración en el servicio de neumología de una institución de salud terciaria. Como antecedentes a destacar, tuvo un episodio de hemoptisis similar cinco meses antes, e historia de tuberculosis en dos de sus hermanos. Adicionalmente, consumía de manera habitual bebidas alcohólicas. 
Ingresó febril, taquicárdico y sin hallazgos signifacativos a la auscultación del tórax. Fue evaluado por el servicio de neumología que no encontró hallazgos preponderantes en el examen del tórax, sin embargo, en la revisión de sus imágenes se ratificó la presencia de una cavitación apical derecha de paredes irregulares con una lesión circunferencial de densidad de tejido blando en su interior, hallazgo que dibujaba el signo del "aire creciente" lo cual condujo a la sospecha de una "bola de hongos". (Ver Figura 1 y 2). Se le realizó una fibrobroncoscopia diagnóstica en donde no se observaron alteraciones estructurales mayores en el árbol bronquial, pero se pudo determinar que la hemoptisis se originaba en el lóbulo superior derecho. Las baciloscopias en esputo fueron negativas para bacilos ácido alcohol resistentes, al igual que el estudio de biología molecular GENEXPERT MTB / RIF y otros estudios microbiológicos, salvo, la presencia de hifas hialinas septadas en la tinción de hidróxido de potasio, $\mathrm{KOH}$. No se encontraron hallazgos clínicos o paraclínicos que configuraran un estado de inmunodeficiencia.

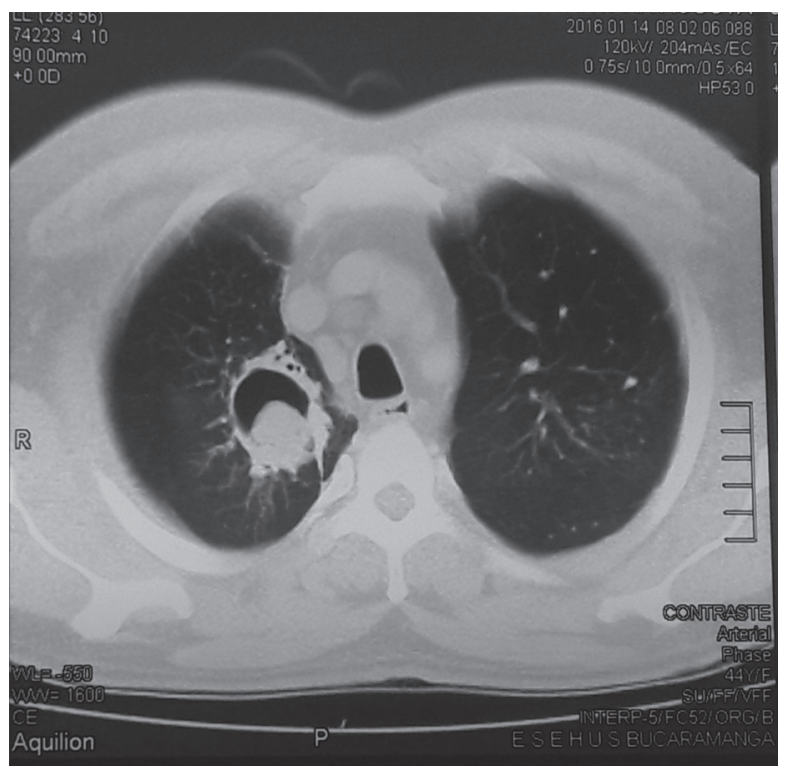

Figura 1. En corte axial de tomografía se observa lesión cavitada con material en su interior sugestivo de bola de hongos Fuente: autor

Se le indicó anfotericina de oxicolato como parte de su manejo pero, no tuvo mayores cambios en su evolución clínica. Por indicación del grupo de neumología se cambió el tratamiento antimicótico a itraconazol oral.

Durante su observación hospitalaria tuvo un descenso importante en los niveles de hemoglobina y hematocrito asociado a la presencia de hemoptisis significativa y persistente. (Ver Gráfica 1). Se solicitó la embolización selectiva de las arterias bronquiales involucradas detectadas por angiografía, como medida transitoria a la lobectomía terapéutica. (Ver Figura 3). Posterior al procedimiento hubo una discreta reducción del sangrado, no obstante, al cabo de 48 horas presentó un episodio de hemoptisis masiva con compromiso hemodinámico que derivó en un procedimiento quirúrgico de emergencia. Se realizó lobectomía superior derecha por toracotomía y se observó una caverna apical sin ruptura ni drenaje al espacio pleural (Ver Figura 4). Su postoperatorio en unidad de cuidado intensivo fue satisfactorio, lográndose su extubación temprana sin complicaciones. Finalmente, el paciente fue trasladado a hospitalización en donde se retiraron los tubos de drenaje del tórax. El estudio histopatológico (tinción básica de hematoxilina eosina y plata metenamina) confirmó la presencia de Aspergillus spp. (Ver Figura 5).

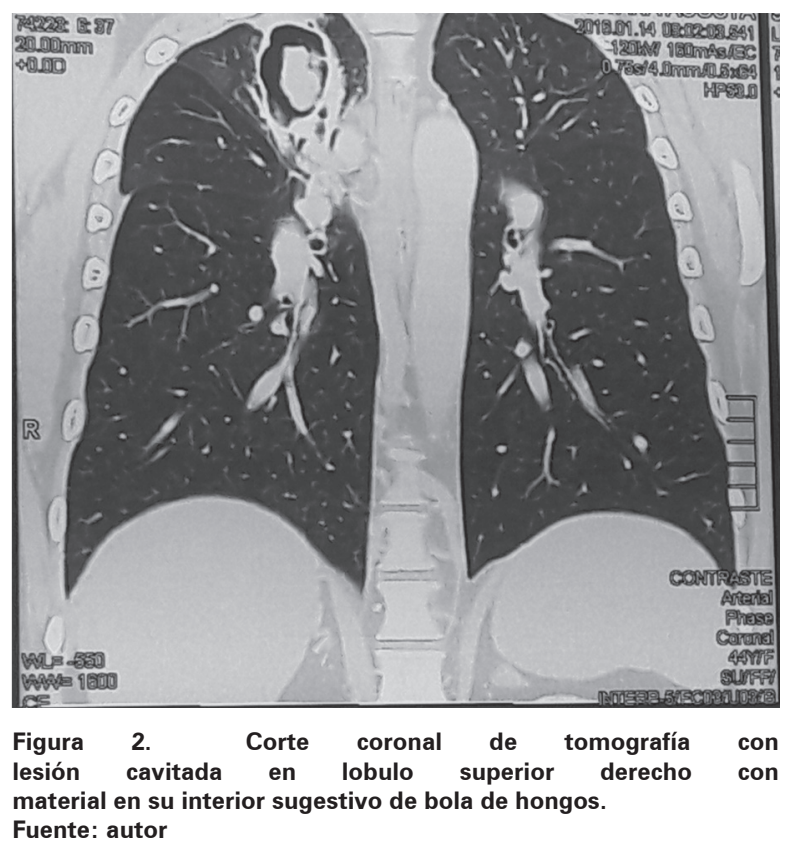

Gráfica 1. Comportamiento de los niveles de hemoglobina y hematocrito durante la hospitalización

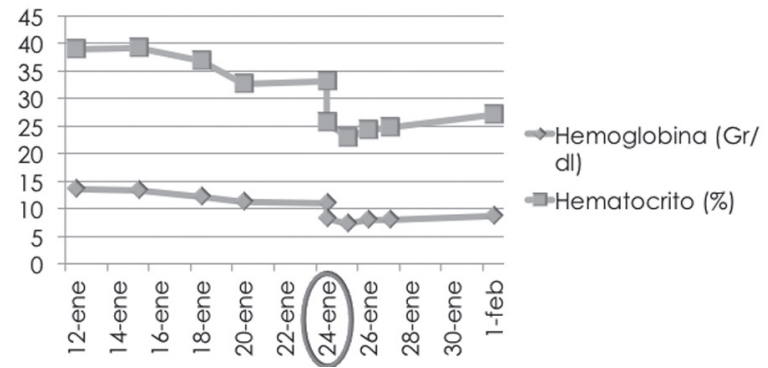

Fuente: autor 
ENERO - ABRIL

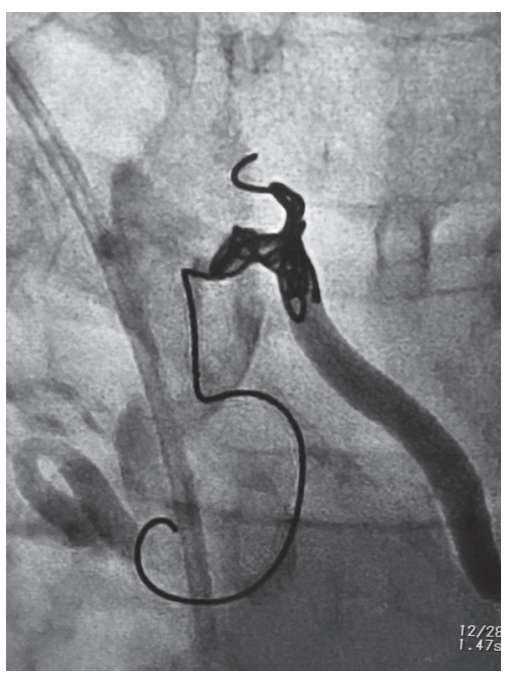

Figura 3. Imagen de embolización selectiva de vasos neoformados a nível de lóbulo superior derecho con microparticulas y coils.

Fuente: Dr Joaquin Gonzales. Departamento de Radiología Intervencionista. Hospital Universitario de Santander.

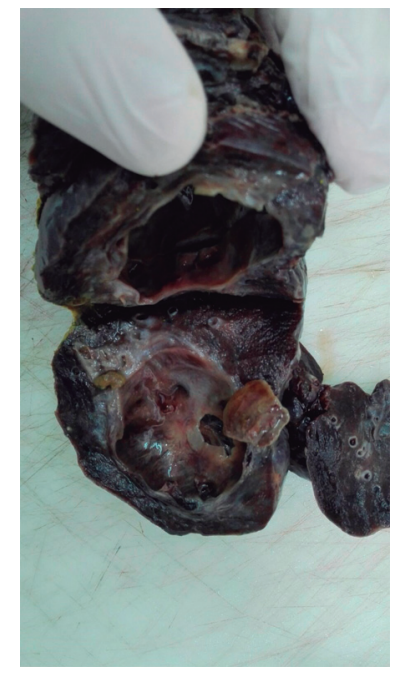

Figura 4. Pieza quirúgica de lobectomía superior derecha en la que se observa lesión cavitada.

Fuente: Departamento de Patología. Universidad Industrial de Santander.

En el caso presentado el manejo quirúrgico de urgencia fue necesario por hemoptisis masiva luego de una embolización arterial sin complicaciones. El tratamiento fue exitoso, lográndose la compensación clínica, la resección de la lesión y el estudio histopatológico pulmonar que permitió la confirmación de la APC. El paciente no requirió intervenciones adicionales posteriores a la resección lobar y su evolución clínica fue satisfactoria luego de unos meses de observación.

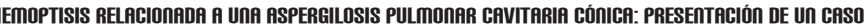

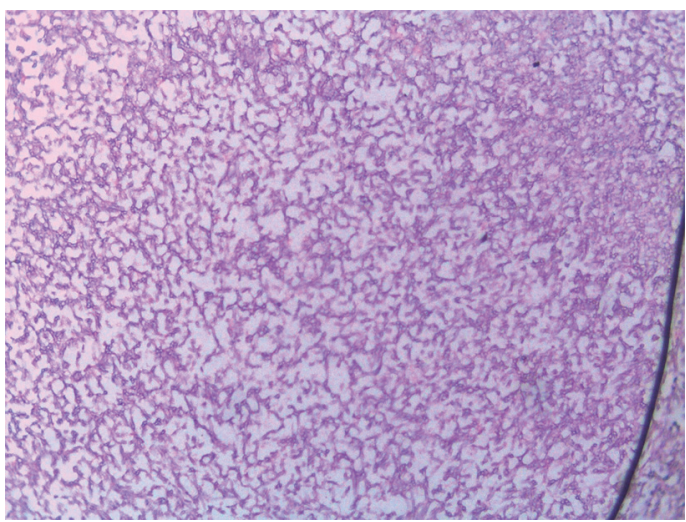

Figura 5. Con tinción con hematoxilina-eosina se observa una bola fúngica compuesta por numerosas hifas septadas dispuestas de manera radial, localizadas en la luz de luces bronquiales dilatadas y en la luz de los vasos formando trombos micóticos. Hallazgos morfológicos compatibles con Aspergillus spp.

Fuente: Departamento de patología. Universidad Industrial de Santander.

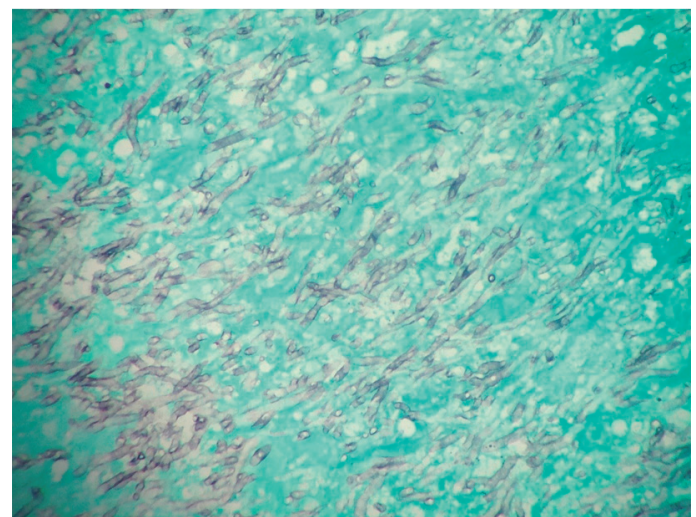

Figura 6. Tinción de plata metenamina en la que se aprecian hifas septadas con ramificaciones a $\mathbf{4 5}$ grados sin conidias ni clamidiosporas que se disponen radialmente.

Fuente: Departamento de patología. Universidad Industrial de Santander.

\section{Discusión}

La aspergilosis pulmonar crónica se ha descrito con una amplia variedad de presentación, desde el aspergiloma simple hasta la aspergilosis fibrosante crónica, en cuanto a la variable observada en el caso, se encontró que es un claro ejemplo de una APCC la cual, en general, muestra múltiples cavidades que pueden contener o no un aspergiloma, ocasionando síntomas sistémicos y pulmonares durante al menos tres meses de seguimiento. En la literatura revisada no se evidencia una prevalencia mayor de una u otra variedad, pues esto depende de los factores de riesgo y el estado de inmunidad innato de cada paciente ${ }^{6}$. 
Las alteraciones del parénquima pulmonar se describen como factor de riesgo para la colonización pulmonar por aspegilus, entre ellas, las secuelas secundarias a tuberculosis pulmonar hasta en un $22 \%$, lo que se evidencia en este caso. Por lo anterior, este diagnóstico debe ser sospechado y descartado en paciente con síntomas respiratorios y antecedente de daño estructural del parénquima pulmonar. Cuervo y cols. describen que las lesiones por aspergillus se presentan preponderantemente en los lóbulos superiores y pueden ser únicas o múltiples7, lo cual coincide con los hallazgos imagenológicos y anatomopatológicos de este caso.

La presentación clínica de esta patología es inespecífica y puede ser confundida con otros agentes etiológicos, lo que se observa en este caso, en el que se presenta con síntomas respiratorios inespecíficos y un episodio de hemoptisis; series de casos describen una presentación clínica de aspergilosis con hemoptisis hasta en un $70 \%$, en menor proporción tos, fiebre y dificultad respiratoria ${ }^{10,11}$.

Se cuenta con una variedad de métodos diagnósticos de esta patología, entre los que se encuentra la medición de antígeno galactomanano en suero y lavado broncoalveolar, con una sensibilidad variable y una especificidad cercana al 80\%; sin embargo, el uso de betalactámicos, entre otras causas, puede generar falsos positivos7. El uso previo de betalactámicos en este paciente fue un motivo que llevó a no realizar dicho estudio, inclinando el abordaje diagnóstico al lavado broncoalveolar con resultados inespecíficos.

Las complicaciones pueden aparecer en el curso de la enfermedad, siendo la hemoptisis una de las más temidas por el riesgo de compromiso vital del paciente con APCC, con una mortalidad que oscila entre el 7-30\%,12 El presente caso es un claro ejemplo de hemoptisis masiva con compromiso sistémico en un paciente con diagnóstico clínico y radiológico de aspergilosis pulmonar. Se describe la diseminación hematógena en pacientes con inmunocompromiso marcado como otra complicación, la cual no se presenta en este caso, pues no se documentó inmunosupresión ${ }^{12}$.

Dentro del manejo de la aspergilosis pulmonar cavitaria se plantea el uso de antimicóticos, entre estos el itraconazol es el medicamento de elección, el cual fue escogido para el tratamiento médico del paciente, ya que revisiones han encontrado buenas concentraciones en el seno del aspergiloma cuando se usan dosis de 100 - 200 mg/día; esto se logra por la alta penetración a través de la pared de la caverna y la buena concentración en material purulento. Por el contrario, por la poca vascularización del aspergiloma no se logra buena penetración de otros antimicóticos como la anfotericina, y esta alternativa terapéutica se reserva para aspergilosis invasiva ${ }^{13}$.

En el caso descrito se presenta una APCC complicada por una hemoptisis masiva recurrente que requirió manejo antimicótico y embolización selectiva de las arterias bronquiales como terapia puente para la resección quirúrgica de la lesión ${ }^{14}$. Las guías clínicas para el manejo de hemoptisis masiva incluyen en su algoritmo de tratamiento la embolización selectiva de arterias bronquiales en pacientes inestables hemodinámicamente en caso de localizar mediante angiografía el sitio de sangrado ${ }^{15,16}$; para la hemoptisis severa específicamente en el contexto de aspergilosis pulmonar está indicado el manejo como terapia puente con embolización selectiva de arterias bronquiales de cara a la cirugía. En el trabajo realizado por $\mathrm{Corr}^{17}$, se logró concluir que la embolización bronquial es efectiva para tratar hemoptisis aguda severa secundaria a una aspergilosis cavitada, permitiendo estabilizar al paciente para el manejo quirúrgico definitivo; en la embolización de arterias bronquiales se ha descrito un porcentaje de éxito, que oscila entre el 50-90\%2,18, sin embargo, la tasa de recidiva no es despreciable y compromete alrededor de la cuarta parte de los pacientes afectados ${ }^{13}$.

Lo anterior se pudo observar en el presente caso, ya que se presentó una recidiva del sangrado de forma masiva, lo que generó inestabilidad hemodinámica y respiratoria. Existen otras medidas descritas frente a pacientes con hemoptisis masiva dentro de las que se incluyen la intubación selectiva y el taponamiento endobronquial, las cuales también son medidas temporales que buscan estabilizar al paciente de para ser llevado al tratamiento quirúrgico, que de lograrse la resección completa de la lesión sin inoculación de elementos al espacio pleural suele ser curativa ${ }^{2}$. En este caso se implementó la intubación selectiva y de inmediato se realizó manejo quirúrgico con lobectomía superior derecha, la cual logró el control del sangrado y resolución de la enfermedad.

Las limitaciones de este trabajo corresponden a las propias de los estudios de casos, los cuales proceden de las prácticas y experiencias clínicas individuales donde el contexto clínico hace que la aplicación de las mismas aquí expuestas deban ser individualizadas. 


\section{CONCLUSIONES}

La APC es una enfermedad con un espectro de presentación amplio lo que puede llevar a un abordaje diagnóstico inadecuado; algunas de sus variantes se han asociado a patologías que ocasionan cambios estructurales pulmonares, como es el caso de la tuberculosis, que es muy frecuente en este medio.

La detección y manejo de complicaciones de la APCC permite evitar la progresión de la enfermedad impidiendo un mayor daño pulmonar y la aparición de hemoptisis masiva que puede poner en riesgo la vida del paciente.

Aún hay discrepancias sobre el mejor tratamiento antifúngico, su efectividad y su papel en el tratamiento de la APC. Se requieren más estudios que permitan realizar recomendaciones con un mayor peso estadístico. Sin embargo, el uso de triazoles como el itraconazol, puede contemplarse en el tratamiento de mantenimiento de la APCC con el fin de mejorar la calidad de vida y reducir la intensidad de los síntomas.

La embolización arterial bronquial selectiva es la medida de rescate inicial frente a los casos de hemoptisis masiva y permite la planeación de un procedimiento curativo mayor.

El manejo quirúrgico sin duda es la estrategia de manejo definitivo para la APCC, ya que permite un rápido control de los síntomas, prevención de complicaciones y en la mayoría de los casos, la curación del proceso infeccioso pulmonar.

\section{Agradecimientos}

Extensos agradecimientos al Hospital Universitario de Santander y su departamento de radiología intervencionista liderado por el Dr. Joaquin Gonzales por su valiosa colaboración en el manejo del paciente y al departamento de Patología de la Universidad Industrial de Santander por su aporte en la realización y documentación del dignóstico histopatológico del presente caso.

\section{Consideraciones Éticas}

El presente trabajo corresponde a un reporte de caso por lo que no implica riesgo inherente a la intervención en los individuos incluidos. Según lo que establece la resolución colombiana clasifica como un estudio sin riesgo pues no realiza ninguna intervención o modificación intencional en ninguna variable sobre el paciente incluido. Se solicitó consentimiento informado al paciente quien aceptó la publicación del caso para fines científicos.

\section{FUENTES DE FINANCIACIÓN}

Ninguno de los autores que participaron en el presente trabajo obtuvo apoyo financiero durante el tiempo de realización del mismo a través de entidades ni por medio de vinculaciones con asociaciones sin ánimo de lucro.

\section{Conflictos de INTERÉS}

Los autores declaran no tener conflictos de interés.

\section{Referencias BibLIOGRÁficas}

1. Deening D, Cadranel J, Beigelman C, Ader F, Chakrabarti A, et al. Chronic pulmonary aspergillosis: rationale and clinical guidelines for diagnosis and management. Eur Respir J. <2016; 47:45-68.

2. Izumikawa K. Recent advances in chronic pulmonary aspergilosis. The Japanese Respiratory Society. 2015.

3. Denning D, Pleuvry A, Cole D. Carga global de la aspergilosis pulmonar crónica como una secuela de tuberculosis pulmonar. Boletín de la Organización Mundial de la Salud, Diciembre 2011. [consultado 15 de abr. 2016] Disponible en http://www.who.int/ bulletin/volumes/89/12/11-089441-ab/es/

4. Kosmidis C, Denning DW. The clinical spectrum of pulmonary aspergillosis. Thorax. 2015;70:270-7.

5. Ennett J. On the parasitic vegetable structures found growing in living animals. Trans Royal Soc Edinburgh. 1842;15:277-79.

6. Passera E, Rizzi A, Robustellini M, Rossi G, Della C, et al. Pulmonary aspergilloma. Thoracic Surg Clin. 2012;22:345-61.

7. Cuervo SI, Gómez JC, Rivas P, Guevara FO. Actualización en aspergilosis con énfasis en aspergilosis invasora. Infectio. 2010;14(S2):S131-44.

8. Velez J, Suarez R. Protocolo de estudio y manejo de pacientes con aspergilosis. Infectio. 2012;16:114-17.

9. Lopez A, Berazain C. Invasive pulmonary aspergillosis in neutropenic patient. Gac Med Bol. 2015;38(1):38-42.

10. Curberlo J, Galván JM, Aspa J. Actualización sobre Aspergillus, Pneumocystis y otras micosis pulmonares oportunistas. Arch Bronconeumol. 2015;51(12):647-53.

11. Regnard JF, Icard P, Nicolosi M, Spagiarri L, Magdeleinat P, et al. Aspergilloma: A series of 89 surgical cases. Ann Thorac Surg. 2000;69:898-903

12. Kohno S, Izumikawa K, Ogawa K. Intravenous micafungin versus voriconazole for chronic pulmonary aspergillosis: a multicenter trial in Japan. J Infect. 2010;61:410-18.

13. Martínez J, Márquez M, Sola O, Are J, Grarau J. Tratamiento del aspergiloma pulmonar. Revisión clínica. Enferm Infecc Microbiol Clin. 2000;18:413-5.

14. Lordan JL, Gascoigne A, Corris PA. The pulmonary physician in critical care - Illustrative case 7: Assessment and management of massive haemoptysis. Thorax. 2003;58(9):814-19.

15. Gerard A. A retrospective review comparing the treatment outcomes of emergency lung resection for massive haemoptysis with and without preoperative bronchial artery embolization. Eur J Cardiothorac Surg. 2014;45(2):251-5.

16. Corr P. Management of severe hemoptysis from pulmonary aspergilloma using endovascular embolization. Cardiovasc 
Intervent Radiol. 2006;29(5):807-10.

17. Walsh TJ, Anaisssie EJ, Denning DW, Herbrechht R, Kontoyiannis DP, Marr KA, et al. Tratamiento de la Aspergilosis: Guías para la práctica clínica de la Sociedad de Enfermedades Infecciosas de los
Estados Unidos de América (IDSA). Clinical Infectious Diseases. 2008:46:T1-T36.

18. Fortún J, Meije Y, Fresco G, Moreno S. Aspergilosis. Formas clínicas y tratamiento. Enferm Infecc Microbiol Clin. 2012;30(4):201-208. 